\title{
Robotic Technology for Spinal Cord Injury: Upper Extremity
}

\author{
Berna ÇELIK \\ Istanbul Physical Medicine Rehabilitation Training and Research Hospital, Istanbul, Turkey
}

\begin{abstract}
Spinal cord injury brings a sudden change in the lives of the affected individuals and causes permanent functional motor limitations. Although this condition has been believed to be refractory to treatment for many years, the life expectancy of affected individual is comparable to that of healthy individuals with the application of new treatment methods. Upper extremity function in tetraplegic patients affects the quality of life. Currently, the use of robots has increased for different treatment alternatives. Robot-mediated rehabilitation includes robot assistance, robotic perturbation, adding virtual reality to robot-mediated therapy, and interfacing the brain with a robotic device. The effects of upper extremity robotic rehabilitation, during and following treatment, and their side effects will be discussed along with currently available literature.

Keywords: Spinal cord injury, robot, rehabilitation, upper extremity
\end{abstract}

\section{Introduction}

Traumatic spinal cord injury causes a sudden change in the life of a person and affects their quality of life and ability to have an independent life because of their permanent functional movement restriction; furthermore, in terms of society, it has significant impacts on health services and economy $(1,2)$. Although the sudden damage to the central nervous system was considered to be resistant to treatment for many years, over the last 30 years, this opinion has changed with an improved understanding of the mechanisms of healing and reorganization of the neural pathways (3).

In spinal cord injuries, neurotransmission is subjected to partial or complete interruption at the spinal cord level between muscles and the central nervous system. The messages coming from the brain or surrounding tissues cannot be transmitted, and goal-oriented movements cannot be completely established. The ideal repair of damage at this level is regeneration.
According to the severity of the lesion, motor, sensory, and autonomic functions can spontaneously recover or improve at a certain level (4).

Currently, there are some treatments that provide guidance and support to spontaneous recovery. These treatments are as follows: constraint-induced movement therapy, robotic-assisted therapy, goal-oriented treatments, pharmacological treatments (e.g., selective serotonin reuptake inhibitors), epidural spinal stimulation, non-invasive cortical stimulation, and treatments that use the aforecited treatments together (3).

The purpose of rehabilitation is to improve the control of the remaining muscle strength and to improve the function and quality of life. Repetitive, progressive, and challenging practices using the affected extremity stimulate plastic changes in the neural connections and support motor control and learning. All of these changes, including functional, structural-anatomical, and neurophysiological processes are defined as neuroplasticity. 
Plastic changes might be found through neuraxis regardless of the lesion level and whether the spinal cord injury is complete or incomplete. These changes include changes in the features of the unaffected neuronal pathways, collateral sprouting in unaffected and damaged axons, and synaptic new adjustments (4).

The fastest neurological recovery period is the first 3 months after injury, and functional recovery continues up to 6-12 months. Although individuals with spinal cord injuries who are initially sensory and motor complete have the lowest potential for neurological and functional recovery, individuals who are initially motor incomplete have the possibility to regain a significant ambulation function. Bilateral loss of grasp function among individuals with severe cervical spinal cord injury limits a person's possibility to independently live or work following injury.

Therefore, the priority in these patients is to regain their grasp and reaching functions. If there is enough voluntary muscle control at the distal to the elbow level, surgeries, such as tendon and muscle transfers, tenodesis, and arthrodesis, can be successfully applied to gain meaningful grasp function. If there is no sufficient muscle function below the elbow level or if the patient does not accept surgical treatment, grasp prosthesis based on functional electrical stimulation might be an option in acquiring upper extremity function. For patients with severe spinal cord injury without voluntary upper extremity muscle contraction, aid devices that provide access to the Internet and social media are used. These devices can be computer controlled or mouth, eye, or voice controlled depending on the remaining functions of the individual. For cases that require respiratory aid devices and when it is difficult to use these technologies, studies on new advanced technology computerassisted interface devices continue (5).

With the advancement of technology in rehabilitation programs, robots are now beginning to be part of rehabilitation programs. Robots may provide the repetition of a movement as desired. During robot-assisted therapy in appropriate patients, the movements can be completed and observed by the patients themselves. Therefore, robot-assisted therapies can also increase the motivation of the patient.

According to the application, robot-assisted therapies are grouped under four main headings (Table 1). Despite their reliance on different etiologies, spinal cord injuries and patients with stroke demonstrate improvement with similar cortical reorganization mechanisms. Therefore, the different treatment results performed by robots usually deal with patients with stroke and spinal cord injuries $(3,6)$.

\section{Robot Support}

For patients who cannot perform a movement, the robot either makes or directs the patient to perform the movement. Studies have revealed that robot-assisted wrist movements or grasp movements cause different activities depending on the voluntary movement on the neural cortical connections in healthy individuals $(3,7,8)$. Furthermore, robotic-assisted one-sided wrist movements change the alpha and beta waves in contralat-
Table 1. Robot-assisted rehabilitation applications

$$
\begin{aligned}
& \text { 1- } \text { Robot-assisted therapy } \\
& \text { 2- } \quad \text { Complicating movement with the help of the robot } \\
& \text { 3- } \quad \text { Adding virtual reality to robot-assisted treatment } \\
& \text { 4- } \quad \text { Connecting the brain to a robotic device via an interface }
\end{aligned}
$$

eral cortical areas that participate in voluntary wrist movements $(3,7)$. Thus, active voluntary and seemingly passive robot-assisted motor movements activate similar brain regions (3).

Although neuroplasticity is enhanced by the challenging and progressive motor learning, it is considered that using only repetitive motor movement learning is not as effective as the first one (3). Via active motor learning in the wrist; 1 . In the contralateral primary motor cortex activity, 2 . In motor excitability participation curves, and 3. Compared to passive motor learning intracortical facilitation has shown greater increase $(3,9)$.

The evidence found in the robot treatment for patients with stroke supports the notion that after robot-assisted therapy, reorganization of brain connections is possible in terms of both regional activation and interhemispheric and intrahemispheric functional connections $(3,10,11)$.

\section{Complicating Robotic Movement}

In this application of the robotic treatment program, while performing a target movement, the deterioration of the movement by applying an external force makes the practice difficult. Complicating the movement might create more stimulation to learning by increasing the intensity of training. However, complicating the movement might disrupt the learning process (3).

A general application regulates the arm movement deterioration and a person's adaptation to this by implementing robot-assisted force regions. The deterioration of the general application of robot-assisted arm movement by applying force regulates this area and enables people to adapt. During this adaptation process, corticostriatal neural activation and intracortical inhibition at short intervals and the presence of changes with cortical stimulability accompanied with facilitation have been demonstrated $(3,12,13)$.

Complicating the movement stimulates the adaptation of a healthy motor system. As a response to the deteriorated movement, reactive feedback adjustments occur. Adaptation is a rapid process, and it is different from the learning provided by repeating the same movement $(3,14)$. While there is neuroplasticity in the learning provided by repetition, in adaptation, cerebellar error-based learning occurs $(3,15)$. In adaptation, orientation from corticostriatal activation to cortico-cerebellar adaptation occur $(3,13,16)$. Shift changes of activation in neural connections continue during a period of rest $(3,17)$. This situation supports the existence of the motor memory consolidation process (neuroplasticity). Preliminary studies support that it is beneficial to add error augmentation strategy-based learning to upper extremity rehabilitation in patients with chronic stroke $(3,18,19)$. 
Addition of Virtual Reality to Robot-Assisted Therapy

Virtual reality is based on experiencing visual forms of computer-simulated situations (Figure 1). This situation provides an extremely convenient opportunity for simulating awarding movement. Reward regions of the brain are considered to activate dopaminergic paths in animal models $(3,20)$.

Recent studies support that the motor cortex responds to rewarding the successful behavior of the primary motor cortex, which participates in motor learning in healthy individuals. Whereas motor memory lasts long with rewarding, with punishment, it is short lived. Therefore, for correct movement sequences, strategies in which reward signal sequences are matched with virtual reality might provide clinical benefit $(3,21,22)$.

\section{Interfacing the Brain with a Robotic Device}

Brain computer interfacing can be defined as a means of interacting with computers via thoughts. The purpose of the brain interface device technology is to provide a link between the brain and external devices and to improve the control of individuals in situations such as limb amputation, stroke, and spinal cord injury by enabling a faster and more efficient communication (3). Figure 2 shows a diagram of a brain computer interface mapping (23). The purpose is to use this technology both as an assistive technology and as a rehabilitation device. The main focus of using these in spinal cord injury is to improve communications and environmental control as an assistive or alternative channel in individuals with high levels of personal injury. The combination with neuroprothesis in upper extremity use is promising. However, there is a need for further studies in terms of the side effects that may occur during training (such as neuropathic pain) $(3,5,24)$.

In a study on robotics technologies developed and applied with the upper extremities in $\mathrm{SCl}$ conducted by Cortes et al. (25), 10 chronic traumatic tetraplegic spinal cord injured patients who were included in wrist robotic training programs were followed for 6 weeks ( $1 \mathrm{~h} /$ day, 3 times/week). This study included the patients who had an extensor carpi radialis muscle strength of 1-4 / 5, a duration of injury $>1$ year, and patients who could tolerate sitting in an upright position for at least $1 \mathrm{~h}$ and who could adapt and understand the robotic treatment protocol. Exclusion criteria in the study were patients with progressive neurodegenerative diseases, concomitant head trauma or stroke, uncontrollable pain or exercise intolerance on the affected side, advanced mobility limitation, irreversible muscle contractures, patients who were medically unstable or with drug use that affected the central nervous system, or patients with transcranial magnetic stimulation contraindications (past history of seizures or epilepsy, presence of metal implants in the brain, presence of pacemakers and pregnancy) (25).

Wrist flexion-extension, abduction-adduction, and pronationsupination movements were applied with the InMotion 3.0 wrist robot without support and by applying force or resistance for 3 days a week, for 6 weeks, for a total of 18 sessions. Treatment programs were designed as a maximum of $1 \mathrm{~h}$ of exercise, 1 -min rest between each adaptive treatment regimen, for a total of a

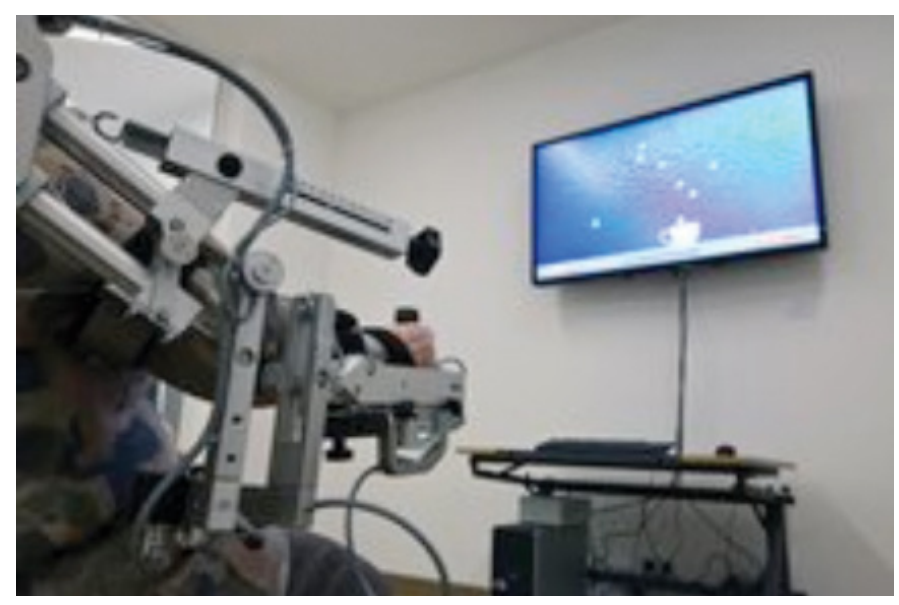

Figure 1 . Virtual reality and robotic upper extremity rehabilitation

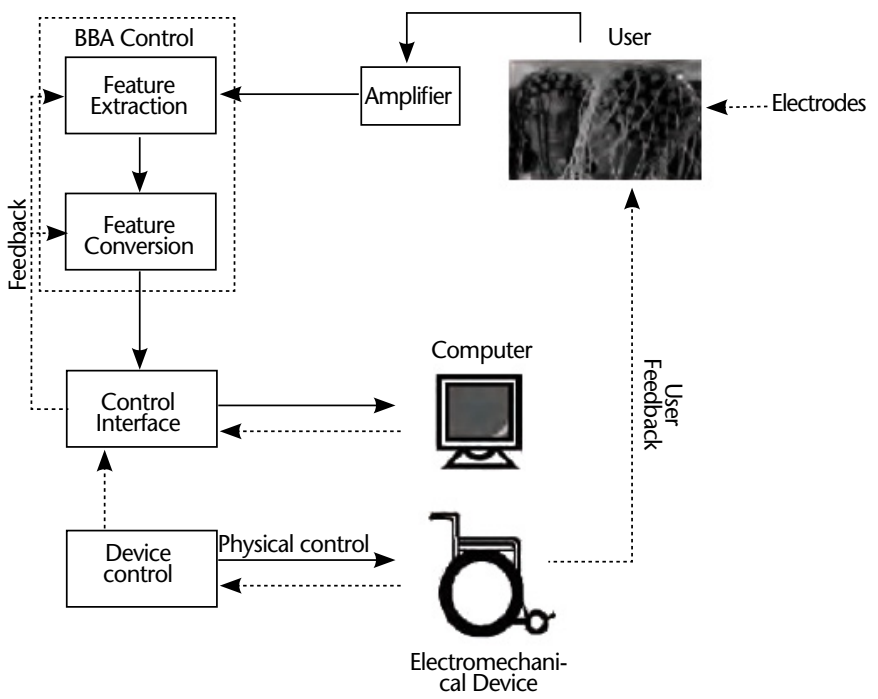

Figure 2. Diagram of the brain computer interface

maximum of 1000 movements. As a follow-up, the results of the cases were assessed with kinematic measurements and neuropsychological result scales, whose clinical result scales were determined with transcranial magnetic stimulation of motor performance. The kinematic movement measurements of the motor performance evaluated: 1 . purpose, 2 . deviation in the movement, 3. average speed, 4 . peak speed, 5 . the mildness of the movement, 6 . the duration of the movement. Furthermore, 1. The upper extremity motor score and 2. the modified Ashworth scale were followed with the clinical result scales, while 3. The pain results were followed with a visual analog scale. The neurophysiological result scales in this study, on the other hand, cover 1. resting motor threshold, 2. motor stimulated potential amplitudes and latencies of rest, and 3. the motor stimulated potential facilitation in this study, all of which were determined with transcranial magnetic stimulation. As a result, after six weeks of robotic therapy, a significant improvement was achieved in reaching the goal of the movement and in softness. However, there was no change in the deviation, average and peak speed, or the duration of the movement. There was 
also no significant enhancement in the upper extremity motor scores for those patients that received treatment and those who did not receive treatment. After the treatment, there was no change in the evaluation of the spasticity levels of the four upper extremity muscles that were assessed, and also, the pain levels did not change. Also no significant change was observed in the neurophysiological parameters that were studied with transcranial magnetic stimulation (25).

The authors stated a number of limitations to their study, such as the fact that the study was conducted with a small number of patients, there was no control group, and in the stroke exercises, there had been more sessions with robotic upper extremity training, and there was an insufficient number of sessions. However, it was stated that spinal cord injured individuals who received robotic upper extremity training demonstrated good compliance, and the application was safe for patients in an environment that can be controlled (25).

Yozbatiran et al. (26) published their study on a 28-year-old female who had had incomplete spinal cord injury (C2 AIS C) 29 months ago and who was included in the upper extremity robot program. The patient underwent $12 \times 3 \mathrm{~h}$ of treatment for four weeks in three different modes: passive, active/assistive, and resistance- with MAXI Exo-II. The purpose of the treatment was to improve the patient's strength and joint mobility spacing with a single joint exercise. The result scales were determined to be: Action Research Arm Test, Jebsen-Taylor Hand Function Test, and the AIS-upper extremity motor score. The safety of the application was assessed with a 5-point scale as fatigue, pain, and discomfort. As a result, although some improvement was observed in the left hand and arm, no change was determined in the right hand or arm function. No side effects of the application, such as increased pain, fatigue, or discomfort, were found. In this case report, an upper extremity robotic rehabilitation robot was evaluated as applicable, safe, and effective (26).

The interest in robotic treatment conducted in the treatment and follow-up of neurological damage is increasing. However, there are gaps concerning the possible side effects and puzzles of this practice. The issues to be clarified in future robotic therapy are (3):

1. The impacts of robotic treatment on neural connections and neuroplasticity are unknown.

2. The effects of complicating the movement in robotic treatment on neuroplasticity in neurologic patients are not known. There is a need to study the correlation and the clinical results of the neurological illnesses of this treatment.

3. In robot-based motor adaptation, the impacts of motivation/reward on neuroplasticity are not known. There is a need for new studies on this subject.

4. It is believed that the reorganization of the brain that occurs with brain interface devices may cause inhibition or may affect learning the new applications by interfering with the learning. Thus, long term repetition may create resistance in learning new movements for related neurons (3).
As a result, as life expectancy among individuals with spinal cord injury increases, the quality of life and rehabilitation programs are becoming increasingly important. Technological advances offer new treatment options in this area (27). Robots are gradually taking an important place among new methods of treatment for spinal cord injuries. In particular, since the development of high-tech imaging methods, the variety of available robots and their technological features will continue to increase in the future, and we will be able to gain a better understanding of the effects, especially on neuroplasticity, of this treatment method. In the future, personalized reproducible, and standardized combined treatments will become increasingly important.

Peer-review: This manuscript was prepared by the invitation of the Editorial Board and its scientific evaluation was carried out by the Editorial Board.

Conflict of Interest: No conflict of interest was declared by the author.

Financial Disclosure: The author declared that this study has received no financial support.

\section{References}

1. Celik B, Gultekin O, Beydogan A, Caglar N. Domain-specific quality of life assesment in spinal cord injured patients. Int J Rehabil Res 2007;30:97-101. [CrossRef]

2. Unalan H, Celik B, Sahin A, Caglar N, Esen S, Karamehmetoglu SS. Quality of life after spinal cord injury: The comparison of the SF-36 health survey an its spinal cord injury-modified version in assesing the health status of people with spinal cord injury. Neurosurg Q 2007; 17:175-9. [CrossRef]

3. Turner DL, Ramos-Murguialday A, Birbaumer N, Hoffman U, Luft A. Neurophysiology of robot-mediated training and therapy: a perspective for future use in clinical populations. Front Neurol 2013;4:184. [CrossRef]

4. Onifer SM, Smith GM, Fouad K. Plasticity after spinal cord injury: relevance to recovery and approaches to facilitate it. Neurotherapeutics 2011;8:283-93. [CrossRef]

5. Rupp R. Challenges in clinical applications of braincomputer interfaces in individuals with spinal cord injury. Front Neuroeng 2014;7:38. [CrossRef]

6. Bui T, Sprigle $S$, Backus D. Upper extremity robotic therapy for individuals with spinal cord injury. Available from: https://smartech. gatech.edu/bitstream/1853/14739/7/handout.pdf. 2007.

7. Formaggio E, Storti SF, Galazzo IB, Gandolfi M, GeroinC, Smania N, et al. Modulation of event-related desynchronization in robot-assisted hand performance: brain oscillatory changes in active, passive and imagined movements. J Neuroeng Rehabil 2013;10:24. [CrossRef]

8. Novakovic V, Sanguineti V. Adaptation to constant-magnitude assistive forces: kinematic and neural correlates. Exp Brain Res 2011;209:425-36. [CrossRef]

9. Lotze M, Braun C, Birbaumer N, Anders S, Cohen LG. Motor learning elicited by voluntary drive. Brain 2003;126:866-72. [CrossRef]

10. Takahashi CD, Der-Yeghiaian L, Le V, Motiwala RR, Cramer SC. Robot-based hand motor therapy after stroke. Brain 2008;131:42537. [CrossRef]

11. Sergi F, Krebs HI, Groissier B, Rykman A, Guglielmelli E, Volpe BT, et al. Predicting efficacy of robot-aided rehabilitation in chronic stroke patients using an MRI robotic device. Conf Proc IEEEb Eng Med Biol Soc 2011;2011:7470-3. [CrossRef] 
12. Hunter T, Sacco P, Turner DL. Changes in excitability of the motor cortex associated with internal modelformation during intrinsic visuomotor learning in the upper arm. J Behav Brain Sci 2011;1:140-52. [CrossRef]

13. Krebs HI, Brashers-Krug T, Raush SL, Savage CR, Hogan N, Rubin $\mathrm{RH}$, et al. Robot-aided functional imaging application to a motor learning study. Human Brain Mapp 1998;6:59-72. [CrossRef]

14. Huang VS, Haith A, Mazzoni P, Krakauer JW. Rethinking motor learning and savings in adaptation paradigms model free memory for successfull actions combines with internal models. Neuron 2011;70:787-801. [CrossRef]

15. Rabe K, Livne O, Gizewski ER, Aurich V, Beck A, Timmann D, et al. Adaptation to visuomotor rotation and force field perturbationis correlated to different brain areas in patients with cerebellar degeneration. J Neurophysiol 2009;101:1961-71. [CrossRef]

16. Doyon J, Bellec P, Amsel R, Penhune V, Monchi O, Carrier J, et al. Contribution of the basal ganglia and functionnally related brain structures to motor learning. Behav Brain Res 2009;199:61-75. [CrossRef]

17. Shadmehr R, Holcomb HH. Neural correlates of motor memory consolidation. Science 1997;277:821-5. [CrossRef]

18. Patton JL, Stoykov ME, Kovic M, Mussa-Ivaldi FA. Evaluation of robotic training forces that either enhance or reduce error in chronic hemiparetic stroke survivors. Exp Brain Res 2006;168:368-83. [CrossRef]

19. Abdollahi F, Case Lazzaro ED, Listenberger M, Kenyon RV, Kovic M, Bogey RA, et al. Error augmentation enhancing arm recovery in individuals with chronic stroke: a randomized crossover design. Neurorehabil Neural Repair 2014;28:120-8. [CrossRef]
20. Hosp JA, Pekanovic A, Rioult-Pedotti MS, Luft AR. Dopaminergic projections from midbrain to primary motor cortex mediate motor skill learning. J Neurosci 2011;31:2481-7. [CrossRef]

21. Kapogiannis D, Campion P, Grafman J, Wassermann EM. Reward-related activity in the human motor cortex. Eur J Neurosci 2008;27:1836-42. [CrossRef]

22. Abe $M$, Shambra $H$, Wassermann EM, Luckenbaug D, Schweighofer $\mathrm{N}$, Cohen LG. Reward improves long-term retention of a motor memory through induction of offline memory gains. Curr Biol 2011;21:557-62. [CrossRef]

23. Argunşah AÖ. Beyinden bilgisayara bir yol: beyin bilgisayar arayüzü. Available from: www.emo.org.tr/ekler/a130f1dc6f0c829_ ek.pdf?dergi $=429$

24. Wang W, Collinger JL, Perz MA, Tyler-Cabara EC, Cohen LG, Birbaumer N, et al. Neural interface technology for rehabilitation: exploiting and promoting neuroplasticity. Phys Med Rehabil Clin N Am 2010;21:157-78. [CrossRef]

25. Cortes M, Elder J, Rykman A, Murray R, Avedissian M, Stampa A, et al. Improved performance in chronic spinal cord injury. NeuroRehabilitation 2013;33:57-65.

26. Yozbatiran N, Beriner J, O'Malley MK, Pehlivan AU, Kadivar Z, Boake $C$, et al. Robotic training and clinical assesment of upper extremity movements after spinal cord injury: a single case report. J Rehabil Med 2012;44:186-8. [CrossRef]

27. Celik B, Ones K, Celik EC, Bugdayci DS, Paker N, Avci C, et al. The effects of using the Internet on the health-related quality of life in people with spinal cord injury: a controlled study. Spinal Cord 2014;52:388-91. [CrossRef] 